\title{
Endpoint boundedness for multilinear commutators of Littlewood-Paley operator
}

Jiasheng Zeng 


\title{
ENDPOINT BOUNDEDNESS FOR MULTILINEAR COMMUTATORS OF LITTLEWOOD-PALEY OPERATORS
}

\author{
JIASHENG ZENG
}

Received September 16, 2010

\begin{abstract}
In this paper, we prove endpoint boundedness of multilinear commutators of LittlewoodPaley operators.
\end{abstract}

2000 Mathematics Subject Classification: 42B20; 42B25

Keywords: Littlewood-paley operator, multilinear commutator, Hardy spaces, $B M O\left(R^{n}\right)$

\section{INTRODUCTION AND NOTATIONS}

Let $b \in B M O\left(R^{n}\right)$ and let $T$ be the Calderón-Zygmund operator. The commutator $[b, T]$ of $b$ and $T$ is defined by

$$
[b, T](f)(x)=b(x) T(f)(x)-T(b f)(x) .
$$

A classical result of Coifman, Rochberb and Weiss (see [3]) states that the commutator $[b, T]$ is bounded on $L^{p}\left(R^{n}\right),(1<p<\infty)$. In [2] and [5], boundedness properties of the commutators for the extreme values of $p$ are obtained. In this paper, we will introduce the multilinear commutators of Littlewood-Paley operators and prove boundedness properties of the operators in the extreme cases.

First let us introduce some notations (see [1],[4],[8],[9],[10]). Throughout this paper, $Q$ will denote a cube of $R^{n}$ with sides parallel to the axes. For a cube $Q$ and for a locally integrable function $f$, let $f_{Q}=|Q|^{-1} \int_{Q} f(x) d x$ and $f^{\#}(x)=$ $\sup _{x \in Q}|Q|^{-1} \int_{Q}\left|f(y)-f_{Q}\right| d y$. Moreover, $f$ is said to belong to $B M O\left(R^{n}\right)$ if $f^{\#} \in$ $x \in Q$

$L^{\infty}$ and define $\|f\|_{B M O}=\left\|f^{\#}\right\|_{L^{\infty}}$. We also define the central $B M O$ space by $C M O\left(R^{n}\right)$, which is the space of those functions $f \in L_{l o c}\left(R^{n}\right)$ such that

$$
\|f\|_{\text {СMO }}=\sup _{r>1}|Q(0, r)|^{-1} \int_{Q}\left|f(y)-f_{Q}\right| d y<\infty .
$$

It has been known that(see [9])

$$
\|f\|_{\text {СMO }}=\sup _{r>1}|Q(0, r)|^{-1} \int_{Q}\left|f(y)-f_{Q}\right| d y<\infty .
$$


Also, we give the concepts of the atom and $H^{1}$ space. A function $a$ is called as a $H^{1}$ atom if there exists a cube $Q$ such that $a$ is supported on $Q,\|a\|_{L^{\infty}} \leq|Q|^{-1}$ and $\int a(x) d x=0$. It is well known that the Hardy space $H^{1}\left(R^{n}\right)$ can be characterized in terms of the atomic decomposition (see [4], [9]).

Definition 1. Let $0<\delta<n$ and $1<p<n / \delta$. We shall call $B_{p}^{\delta}\left(R^{n}\right)$ the space of those functions $f$ on $R^{n}$ such that

$$
\|f\|_{B_{p}^{\delta}}=\sup _{r>1} r^{-n(1 / p-\delta / n)}\left\|f \chi_{Q(0, r)}\right\|_{L^{p}}<\infty .
$$

Definition 2. Let $\varepsilon>0, n>\delta>0$ and let $\psi$ be a fixed function that satisfies the following properties:

1) $\int \psi(x) d x=0$

2) $|\psi(x)| \leq C(1+|x|)^{-(n+1-\delta)}$,

3) $|\psi(x+y)-\psi(x)| \leq C|y|^{\varepsilon}(1+|x|)^{-(n+1+\varepsilon-\delta)}$ when $2|y|<|x|$;

We denote $\Gamma(x)=\left\{(y, t) \in R_{+}^{n+1}:|x-y|<t\right\}$ and the characteristic function of $\Gamma(x)$ is written as $\chi_{\Gamma(x)}$. Let $\vec{b}=\left(b_{1}, \cdots, b_{m}\right)$ with $b_{j} \in B M O\left(R^{n}\right)$ for $1 \leq j \leq m$. Then the multilinear commutator of our Littlewood-Paley operator is defined by

$$
S_{\delta}^{\vec{b}}(f)(x)=\left[\iint_{\Gamma(x)}\left|F_{t}^{\vec{b}}(f)(x, y)\right|^{2} \frac{d y d t}{t^{n+1}}\right]^{1 / 2},
$$

where

$$
F_{t}^{\vec{b}}(f)(x, y)=\int_{R^{n}} \prod_{j=1}^{m}\left(b_{j}(x)-b_{j}(z)\right) \psi_{t}(y-z) f(z) d z ;
$$

When $m=1$, set

$$
S_{\delta}^{b}(f)(x)=\left[\iint_{\Gamma(x)}\left|F_{t}^{b}(f)(x, y)\right|^{2} \frac{d y d t}{t^{n+1}}\right]^{1 / 2},
$$

where

$$
F_{t}^{b}(f)(x, y)=\int_{R^{n}}(b(x)-b(z)) \psi_{t}(y-z) f(z) d z
$$

and $\psi_{t}(x)=t^{-n+\delta} \psi(x / t)$ for $t>0$. Set $F_{t}(f)(x)=f * \psi_{t}(x)$, we also define

$$
S_{\delta}(f)(x)=\left[\iint_{\Gamma(x)}\left|f * \psi_{t}(x)\right|^{2} \frac{d y d t}{t^{n+1}}\right]^{1 / 2},
$$

which is the Littlewood-Paley operator (see [1][6, 7][10]).

Let $H$ be the Hilbert space $H=\left\{h:\|h\|=\left(\iint_{R_{+}^{n+1}}|h(y, t)|^{2} d y d t / t^{n+1}\right)^{1 / 2}\right.$ $<\infty\}$. Then for each fixed $x \in R^{n}, F_{t}(f)(x, y)$ may be viewed as a mapping from $[0,+\infty)$ to $H$. It is clear that

$$
S_{\delta}(f)(x)=\left\|\chi_{\Gamma(x)} F_{t}(f)(y)\right\| \text { and } S_{\delta}^{\vec{b}}(f)(x)=\left\|\chi_{\Gamma(x)} F_{t}^{\vec{b}}(f)(y)\right\| .
$$


Given a positive integer $m$ and $1 \leq j \leq m$, we denote by $C_{j}^{m}$ the family of all finite subsets $\sigma=\{\sigma(1), \cdots, \sigma(j)\}$ of $\{1, \cdots, m\}$ of $j$ different elements. For $\sigma \in C_{j}^{m}$, set $\sigma^{c}=\{1, \cdots, m\} \backslash \sigma$. For $\vec{b}=\left(b_{1}, \cdots, b_{m}\right)$ and $\sigma=\{\sigma(1), \cdots, \sigma(j)\} \in C_{j}^{m}$, set $\vec{b}_{\sigma}=\left(b_{\sigma(1)}, \cdots, b_{\sigma(j)}\right), b_{\sigma}=b_{\sigma(1)} \cdots b_{\sigma(j)}$ and set

$\left\|\vec{b}_{\sigma}\right\|_{B M O}=\left\|b_{\sigma(1)}\right\|_{B M O} \cdots\left\|b_{\sigma(j)}\right\|_{B M O}$.

\section{THEOREMS AND PROOFS}

We begin with a few preliminary lemmas.

Lemma 1. Let $1<r<\infty, b_{j} \in B M O\left(R^{n}\right)$ for $j=1, \cdots, k$ and $k \in N$. Then we have

and

$$
\frac{1}{|Q|} \int_{Q} \prod_{j=1}^{k}\left|b_{j}(y)-\left(b_{j}\right)_{Q}\right| d y \leq C \prod_{j=1}^{k}\left\|b_{j}\right\|_{B M O}
$$

$$
\left(\frac{1}{|Q|} \int_{Q} \prod_{j=1}^{k}\left|b_{j}(y)-\left(b_{j}\right)_{Q}\right|^{r} d y\right)^{1 / r} \leq C \prod_{j=1}^{k}\left\|b_{j}\right\|_{B M O} .
$$

Lemma 2. Let $0<\delta<n, 1<p<n / \delta$ and $1 / q=1 / p-\delta / n$. Then $S_{\delta}$ is bounded from $L^{p}\left(R^{n}\right)$ to $L^{q}\left(R^{n}\right)$.

Theorem 1. Let $0<\delta<n$ and $\vec{b}=\left(b_{1}, \cdots, b_{m}\right)$ with $b_{j} \in B M O\left(R^{n}\right)$ for $1 \leq j \leq$ m. Then $S_{\delta}^{\vec{b}}$ is bounded from $L^{n / \delta}\left(R^{n}\right)$ to $B M O\left(R^{n}\right)$.

Proof of Theorem 1. It is necessary only to prove that there exist a constant $C_{Q}$ such that

$$
\frac{1}{|Q|} \int_{Q}\left|S_{\delta}^{\vec{b}}(f)(x)-C_{Q}\right| d x \leq C|| f \|_{L^{n / \delta}}
$$

Fix a cube $Q=Q\left(x_{0}, r\right)$, we decompose $f$ into $f=f_{1}+f_{2}$ with $f_{1}=f \chi_{Q}, f_{2}=$ $f \chi\left(R^{n} \backslash Q\right)$.

When $m=1$, set $\left(b_{1}\right)_{Q}=|Q|^{-1} \int_{Q} b_{1}(y) d y$, we have

$$
\begin{gathered}
F_{t}^{b_{1}}(f)(x, y) \\
=\left(b_{1}(x)-\left(b_{1}\right)_{Q}\right) F_{t}(f)(y)-F_{t}\left(\left(b_{1}-\left(b_{1}\right)_{Q}\right) f_{1}\right)(y)-F_{t}\left(\left(b_{1}-\left(b_{1}\right)_{Q}\right) f_{2}\right)(y),
\end{gathered}
$$

So

$$
\begin{aligned}
& \left|S_{\delta}^{b_{1}}(f)(x)-S_{\delta}\left(\left(\left(b_{1}\right)_{Q}-b_{1}\right) f_{2}\right)\left(x_{0}\right)\right| \\
& =\left|\left\|\chi_{\Gamma(x)} F_{t}^{b_{1}}(f)(x, y)\right\|-\left\|\chi_{\Gamma\left(x_{0}\right)} F_{t}\left(\left(\left(b_{1}\right)_{Q}-b_{1}\right) f_{2}\right)(y)\right\|\right| \\
& \leq\left\|\chi_{\Gamma(x)} F_{t}^{b_{1}}(f)(x, y)-\chi_{\Gamma\left(x_{0}\right)} F_{t}\left(\left(\left(b_{1}\right)_{Q}-b_{1}\right) f_{2}\right)(y)\right\| \\
& \leq\left\|\chi_{\Gamma(x)}\left(b_{1}(x)-\left(b_{1}\right)_{Q}\right) F_{t}(f)(y)\right\|+\left\|\chi_{\Gamma(x)} F_{t}\left(\left(\left(b_{1}\right)_{Q}-b_{1}\right) f_{1}\right)(y)\right\|
\end{aligned}
$$




$$
\begin{aligned}
& +\left\|\chi_{\Gamma(x)} F_{t}\left(\left(b_{1}-\left(b_{1}\right)_{Q}\right) f_{2}\right)(y)-\chi_{\Gamma\left(x_{0}\right)} F_{t}\left(\left(b_{1}-\left(b_{1}\right)_{Q}\right) f_{2}\right)(y)\right\| \\
& =A(x)+B(x)+C(x)
\end{aligned}
$$

For $A(x)$, set $1<p<n / \delta, 1 / q=1 / p-\delta / n$ and $1 / q+1 / q^{\prime}=1$. By Hölder's inequality and Lemma 1,2, we have

$$
\begin{aligned}
\frac{1}{Q} \int_{Q}|A(x)| d x \leq & \left(\frac{1}{|Q|} \int_{Q}\left|b_{1}(x)-\left(b_{1}\right)_{Q}\right|^{q^{\prime}} d x\right)^{1 / q^{\prime}} \\
& \left(\frac{1}{|Q|} \int_{R^{n}}\left|S_{\delta}(f)(x)\right|^{q} \chi_{Q}(x) d x\right)^{1 / q} \\
\leq & C\left\|b_{1}\right\|_{B M O} \frac{1}{|Q|^{q}}\left(\int_{R^{n}}|f(x)|^{p} \chi_{Q}(x) d x\right)^{1 / p} \\
\leq & C\left\|b_{1}\right\|_{B M O} \frac{1}{|Q|^{q}}\|f\|_{L^{n / \delta}}|Q|^{(1-\delta p / n) p} \\
\leq & C\left\|b_{1}\right\|_{B M O}\|f\|_{L^{n / \delta}} .
\end{aligned}
$$

For $B(x)$, take $1<r<n / \delta$ and $1 / s=1 / r-\delta / n$, then by Hölder's inequality we have

$$
\begin{aligned}
\frac{1}{|Q|} \int_{Q}|B(x)| d x & \leq\left(\frac{1}{|Q|} \int_{R^{n}}\left(S_{\delta}\left(\left(b_{1}(x)-\left(b_{1}\right)_{Q}\right) f_{1}\right)(x)\right)^{s} d x\right)^{1 / s} \\
& \leq C|Q|^{-1 / s}\left\|\left(b_{1}(x)-\left(b_{1}\right) Q\right) f \chi Q\right\|_{L^{r}} \\
& \leq C\left(\frac{1}{|Q|} \int_{Q}\left|b_{1}(x)-\left(b_{1}\right)_{Q}\right|^{s} d x\right)^{1 / s}\|f\|_{L^{n / \delta}} \\
& \leq C\left\|b_{1}\right\|_{B M O}\|f\|_{L^{n / \delta}} .
\end{aligned}
$$

For $C(x)$, we have

$$
\begin{gathered}
C(x) \\
\leq\left[\iint_{R_{+}^{n+1}}\left(\int_{Q^{c}}\left|\chi_{\Gamma(x)}-\chi_{\Gamma\left(x_{0}\right)}\right|\left|b_{1}(z)-\left(b_{1}\right)_{Q} \| \psi_{t}(y-z)\right||f(z)| d z\right)^{2} \frac{d y d t}{t^{n+1}}\right]^{1 / 2} \\
\leq C \int_{Q^{c}}\left|b_{1}(z)-\left(b_{1}\right)_{Q}\right||f(z)| \mid \iint_{|x-y| \leq t} \frac{t^{1-n} d y d t}{(t+|y-z|)^{2 n+2-2 \delta}} \\
-\left.\iint_{\left|x_{0}-y\right| \leq t} \frac{t^{1-n} d y d t}{(t+|y-z|)^{2 n+2-2 \delta}}\right|^{1 / 2} d z \\
\leq C \int_{Q^{c}}\left|b_{1}(z)-\left(b_{1}\right)_{Q}\right||f(z)|
\end{gathered}
$$




$$
\begin{gathered}
\times\left(\iint_{|y| \leq t,|x+y-z| \leq t} \mid \frac{1}{(t+|x+y-z|)^{2 n+2-2 \delta}}\right. \\
\left.-\frac{1}{\left(t+\left|x_{0}+y-z\right|\right)^{2 n+2-2 \delta}} \mid \frac{d y d t}{t^{n-1}}\right)^{1 / 2} d z \\
\leq \int_{Q^{c}}\left|b_{1}(z)-\left(b_{1}\right) Q\right||f(z)| \\
\left(\iint_{|y| \leq t,|x+y-z| \leq t} \frac{\left|x-x_{0}\right| t^{1-n}}{(t+|x+y-z|)^{2 n+3-2 \delta}} d y d t\right)^{1 / 2} d z,
\end{gathered}
$$

note that $2 t+|x+y-z| \geq 2 t+|x-z|-|y| \geq t+|x-z|$ when $|y| \leq t$ and

$$
\int_{0}^{\infty} \frac{t d t}{(t+|x-z|)^{2 n+3-2 \delta}}=C|x-z|^{-2 n-1+2 \delta} .
$$

Then, for $x \in Q$,

$$
\begin{aligned}
& C(x) \\
& \leq C \int_{Q^{c}}\left|b_{1}(z)-\left(b_{1}\right)_{Q} \| f(z)\right|\left|x-x_{0}\right|^{1 / 2} \\
& \left(\iint_{|y| \leq t} \frac{t^{1-n} d y d t}{(t+|x-z|)^{2 n+3-2 \delta}}\right)^{1 / 2} d z \\
& \leq C \int_{Q^{c}}\left|b_{1}(z)-\left(b_{1}\right)_{Q} \| f(z)\right|\left|x-x_{0}\right|^{1 / 2} \\
& \left(\int_{0}^{\infty} \frac{t d t}{(t+|x-z|)^{2 n+3-2 \delta}}\right)^{1 / 2} d z \\
& \leq C \int_{Q^{c}}\left|b_{1}(z)-\left(b_{1}\right) Q \| f(z)\right| \frac{\left|x_{0}-x\right|^{1 / 2}}{\left|x_{0}-z\right|^{n+1 / 2-\delta}} d z \\
& \leq C \sum_{k=0}^{\infty} \int_{2^{k+1} Q \backslash 2^{k} Q}\left|b_{1}(z)-\left(b_{1}\right) Q \| f(z)\right| \frac{\left|x_{0}-x\right|^{1 / 2}}{\left|x_{0}-z\right|^{n+1 / 2-\delta}} d z \\
& \leq C \sum_{k=1}^{\infty} 2^{-k / 2}\left(\frac{1}{\left|2^{k} Q\right|} \int_{2^{k} Q}\left|b_{1}(z)-\left(b_{1}\right)_{Q}\right|^{n /(n-\delta)} d z\right)^{(n-\delta) / n} \\
& \left(\int_{2^{k} Q}|f(z)|^{n / \delta} d z\right)^{\delta / n} \\
& \leq C \sum_{k=1}^{\infty} k 2^{-k / 2}\left\|b_{1}\right\|_{B M O}\|f\|_{L^{n / \delta}} \\
& \leq C\left\|b_{1}\right\|_{B M O}\|f\|_{L^{n / \delta}} .
\end{aligned}
$$


When $m>1$, set $\vec{b}_{Q}=\left(\left(b_{1}\right)_{Q}, \cdots,\left(b_{m}\right)_{Q}\right) \in R^{n}$, where $\left(b_{j}\right)_{Q}=|Q|^{-1} \int_{Q} b_{j}(y) d y, 1 \leq j \leq m$. Then we have

$$
\begin{aligned}
F_{t}^{\vec{b}}(f)(x, y) & =\left(b_{1}(x)-\left(b_{1}\right)_{Q}\right) \cdots\left(b_{m}(x)-\left(b_{m}\right)_{Q}\right) F_{t}(f)(y) \\
+ & (-1)^{m} F_{t}\left(\left(b_{1}-\left(b_{1}\right)_{Q}\right) \cdots\left(b_{m}-\left(b_{m}\right)_{Q}\right) f\right)(y) \\
+ & \sum_{j=1}^{m-1} \sum_{\sigma \in C_{j}^{m}}(-1)^{m-j}\left(\vec{b}(x)-\vec{b}_{Q}\right)_{\sigma} \int_{R^{n}}\left(\vec{b}(z)-\vec{b}_{Q}\right)_{\sigma^{c}} \psi_{t}(y-z) f(z) d z \\
= & \left(b_{1}(x)-\left(b_{1}\right)_{Q}\right) \cdots\left(b_{m}(x)-\left(b_{m}\right)_{Q}\right) F_{t}(f)(y) \\
+ & (-1)^{m} F_{t}\left(\left(b_{1}-\left(b_{1}\right)_{Q}\right) \cdots\left(b_{m}-\left(b_{m}\right)_{Q}\right) f_{1}\right)(y) \\
+ & (-1)^{m} F_{t}\left(\left(b_{1}-\left(b_{1}\right)_{Q}\right) \cdots\left(b_{m}-\left(b_{m}\right)_{Q}\right) f_{2}\right)(y) \\
& +\sum_{j=1}^{m-1} \sum_{\sigma \in C_{j}^{m}}(-1)^{m-j}\left(\vec{b}(x)-\vec{b}_{Q}\right)_{\sigma} F_{t}\left(\left(\vec{b}-\vec{b}_{Q}\right)_{\sigma^{c}} f\right)(x, y),
\end{aligned}
$$

thus

$$
\begin{aligned}
& \left|S_{\delta}^{\vec{b}}(f)(x)-S_{\delta}\left(\left(\left(b_{1}\right)_{Q}-b_{1}\right) \cdots\left(\left(b_{m}\right)_{Q}-b_{m}\right) f_{2}\right)\left(x_{0}\right)\right| \\
& \leq\left\|\chi_{\Gamma(x)} F_{t}^{\vec{b}}(f)(x, y)-\chi_{\Gamma\left(x_{0}\right)} F_{t}\left(\left(\left(b_{1}\right)_{Q}-b_{1}\right) \cdots\left(\left(b_{m}\right)_{Q}-b_{m}\right) f_{2}\right)(y)\right\| \\
& \leq\left\|\chi_{\Gamma(x)}\left(b_{1}(x)-\left(b_{1}\right)_{Q}\right) \cdots\left(b_{m}(x)-\left(b_{m}\right)_{Q}\right) F_{t}(f)(y)\right\| \\
& +\sum_{j=1}^{m-1} \sum_{\sigma \in C_{j}^{m}}\left\|\chi_{\Gamma(x)}\left(\vec{b}(x)-\vec{b}_{Q}\right)_{\sigma} F_{t}\left(\left(\vec{b}-\vec{b}_{Q}\right)_{\sigma^{c}} f\right)(x, y)\right\| \\
& +\left\|\chi_{\Gamma(x)} F_{t}\left(\left(b_{1}-\left(b_{1}\right)_{Q}\right) \cdots\left(b_{m}-\left(b_{m}\right)_{Q}\right) f_{1}\right)(y)\right\| \\
& +\left\|\chi_{\Gamma(x)} F_{t}\left(\prod_{j=1}^{m}\left(b_{j}-\left(b_{j}\right)_{Q}\right) f_{2}\right)(y)-\chi_{\Gamma\left(x_{0}\right)} F_{t}\left(\prod_{j=1}^{m}\left(b_{j}-\left(b_{j}\right)_{Q}\right) f_{2}\right)(y)\right\| \\
& =I_{1}(x)+I_{2}(x)+I_{3}(x)+I_{4}(x) .
\end{aligned}
$$

For $I_{1}(x)$, by taking $1<p<n / \delta$ and $1 / q=1 / p-\delta / n$, and by Hölder's inequality and Lemma 1,2, we have

$$
\begin{aligned}
& \frac{1}{|Q|} \int_{Q} I_{1}(x) d x \\
& \leq\left(\frac{1}{|Q|} \int_{Q}\left|\prod_{j=1}^{m}\left(b_{j}(x)-\left(b_{j}\right)_{Q}\right)\right|^{q^{\prime}} d x\right)^{1 / q^{\prime}}\left(\frac{1}{|Q|} \int_{Q}\left|S_{\delta}(f)(x)\right|^{q} d x\right)^{1 / q} \\
& \leq C|| \vec{b}\left\|_{B M O}|Q|^{-1 / q}\left(\int_{Q}|f(x)|^{p} d x\right)^{1 / p} \leq C\right\| \vec{b}\left\|_{B M O}\right\| f \|_{L^{n / \delta}} .
\end{aligned}
$$


For $I_{2}(x)$, if we take $1<p<n / \delta$ and $1 / q=1 / p-\delta / n$, then

$$
\begin{aligned}
& \frac{1}{|Q|} \int_{Q} I_{2}(x) d x \\
& \leq \sum_{j=1}^{m-1} \sum_{\sigma \in C_{j}^{m}} \frac{1}{|Q|} \int_{Q}\left|\left(b \overrightarrow{(x)}-\vec{b}_{Q}\right)_{\sigma} \| S_{\delta}\left(\left(\vec{b}-\vec{b}_{Q}\right)_{\sigma^{c}} f\right)(x)\right| d x \\
& \leq C \sum_{j=1}^{m-1} \sum_{\sigma \in C_{j}^{m}}\left(\frac{1}{|Q|} \int_{Q}\left|\left(\vec{b}(x)-\vec{b}_{Q}\right)_{\sigma}\right|^{q^{\prime}} d x\right)^{1 / q^{\prime}} \\
& \left.\leq\left. C \sum_{j=1}^{m-1}\left\|\vec{b}_{\sigma}\right\|_{B M O}|Q|^{1 / q}\left(\int_{R^{n}} \mid\left(b(x)-\vec{b}_{Q}\right)_{\sigma^{c}}\right) f(x)\right|^{p} \chi Q d x\right)^{1 / q} \\
& \left.\left.\leq C \sum_{j=1}^{m-1} \sum_{\sigma \in C_{j}^{m}}\left\|\vec{b}_{\sigma}\right\|_{B M O}\left(\frac{1}{|Q|} \int_{Q}\left|\left(\vec{b}(x)-\vec{b}_{Q}\right)_{\sigma}\right|^{q} d x\right)^{1 / q}\|f\|_{L^{n / \delta}}\right)\left.(x)\right|^{q} d x\right)^{1 / q} \\
& \leq C \sum_{j=1}^{m-1} \sum_{\sigma \in C_{j}^{m}}\left\|\vec{b}_{\sigma}\right\|_{B M O}\left\|\vec{b}_{\sigma^{c}}\right\|_{B M O}\|f\|_{L^{n / \delta}} \\
& \leq C|| \vec{b}\left\|_{B M O}\right\| f \|_{L^{n / \delta} .}
\end{aligned}
$$

For $I_{3}(x)$, take $1<p<n / \delta$ and $1 / q=1 / p-\delta / n$, so we get

$$
\begin{aligned}
& \frac{1}{|Q|} \int_{Q} I_{3}(x) d x \\
& \leq\left(\frac{1}{|Q|} \int_{Q}\left|S_{\delta}\left(\left(b_{1}-\left(b_{1}\right)_{Q}\right) \cdots\left(b_{m}-\left(b_{m}\right)_{Q}\right) f_{1}\right)(x)\right|^{q} d x\right)^{1 / q} \\
& \leq C|Q|^{-1 / q} \|\left(\left(b_{1}(x)-\left(b_{1}\right)_{Q}\right) \cdots\left(b_{m}(x)-\left(b_{m}\right) Q\right) f_{1}(x) \|_{L^{p}}\right. \\
& \leq C\left(\frac{1}{|Q|} \int_{Q}\left|\left(b_{1}-\left(b_{1}\right)_{Q}\right) \cdots\left(b_{m}-\left(b_{m}\right)_{Q}\right)\right|^{q} d x\right)^{1 / q}\|f\|_{L^{n / \delta}} \\
& \leq C\|\vec{b}\|_{B M O}\|f\|_{L^{n / \delta}} .
\end{aligned}
$$


For $I_{4}(x)$, similary as in the proof of $C(x)$ in Case $m=1$, we have

$$
\begin{aligned}
& I_{4}(x) \leq C \int_{Q^{c}}\left|x_{0}-x\right|^{1 / 2}\left|x_{0}-z\right|^{-(n+1 / 2-\delta)}\left|\prod_{j=1}^{m}\left(b_{j}(z)-\left(b_{j}\right)_{Q}\right)\right||f(z)| d z \\
& \leq C \sum_{k=0}^{\infty} \int_{2^{k+1} Q \backslash 2^{k} Q}\left|x_{0}-x\right|^{1 / 2}\left|x_{0}-z\right|^{-(n+1 / 2-\delta)}\left|\prod_{j=1}^{m}\left(b_{j}(z)-\left(b_{j}\right)_{Q}\right)\right||f(z)| d z \\
& \leq C \sum_{k=0}^{\infty} 2^{-k / 2} \frac{1}{\left|2^{k+1} Q\right|^{1-\delta / n}} \int_{2^{k+1} Q}\left|\prod_{j=1}^{m}\left(b_{j}(z)-\left(b_{j}\right)_{Q}\right)\right||f(z)| d z \\
& \leq C \sum_{k=1}^{\infty} 2^{-k / 2}\left(\frac{1}{\left|2^{k} Q\right|} \int_{2^{k} Q}\left|\prod_{j=1}^{m}\left(b_{j}(y)-\left(b_{j}\right)_{2} Q\right)\right|^{n /(n-\delta)} d y\right)^{(n-\delta) / n}\|f\|_{L^{n / \delta}}
\end{aligned}
$$

$\leq C\|\vec{b}\|_{B M O}\|f\|_{L^{n / \delta}}$.

This completes the proof of Theorem 1 .

Theorem 2. Let $0<\delta<n, 1<p<n / \delta$ and $\vec{b}=\left(b_{1}, \cdots, b_{m}\right)$ with $b_{j} \in B M O\left(R^{n}\right)$ for $1 \leq j \leq m$. Then $S_{\delta}^{\vec{b}}$ is bounded from $B_{p}^{\delta}\left(R^{n}\right)$ to $C M O\left(R^{n}\right)$.

Proof of Theorem 2. It suffices to prove that there exist a constant $C_{Q}$, such that

$$
\frac{1}{|Q|} \int_{Q}\left|S_{\delta}^{\vec{b}}(f)(x)-C_{Q}\right| d x \leq C\|f\|_{B_{p}^{\delta}}
$$

holds for any cube $Q=Q(0, r)$ with $r>1$. Fix a cube $Q=Q(0, r)$ with $r>1$. Set $f_{1}=f \chi_{Q}, f_{2}=f \chi_{R^{n} \backslash Q}$ and $\vec{b}_{Q}=\left(\left(b_{1}\right)_{Q}, \cdots,\left(b_{m}\right)_{Q}\right) \in R^{n}$, where $\left(b_{j}\right)_{Q}=$ $|Q|^{-1} \int_{Q}\left|b_{j}(y)\right| d y, \quad 1 \leq j \leq m$. Then we have

$$
\begin{aligned}
& \left|S_{\delta}^{\vec{b}}(f)(x)-S_{\delta}\left(\left(\left(b_{1}\right)_{Q}-b_{1}\right) \cdots\left(\left(b_{m}\right)_{Q}-b_{m}\right) f_{2}\right)\left(x_{0}\right)\right| \\
& \leq\left\|\chi_{\Gamma(x)}\left(b_{1}(x)-\left(b_{1}\right)_{Q}\right) \cdots\left(b_{m}(x)-\left(b_{m}\right)_{Q}\right) F_{t}(f)(y)\right\| \\
& +\sum_{j=1}^{m-1} \sum_{\sigma \in C_{j}^{m}}\left\|\chi_{\Gamma(x)}\left(\vec{b}(x)-\vec{b}_{Q}\right)_{\sigma} F_{t}\left(\left(\vec{b}-\vec{b}_{Q}\right)_{\sigma^{c}} f\right)(x, y)\right\| \\
& +\left\|\chi_{\Gamma(x)} F_{t}\left(\left(b_{1}-\left(b_{1}\right)_{Q}\right) \cdots\left(b_{m}-\left(b_{m}\right)_{Q}\right) f_{1}\right)(y)\right\| \\
& +\left\|\chi_{\Gamma(x)} F_{t}\left(\prod_{j=1}^{m}\left(b_{j}-\left(b_{j}\right)_{Q}\right) f_{2}\right)(y)-\chi_{\Gamma\left(x_{0}\right)} F_{t}\left(\prod_{j=1}^{m}\left(b_{j}-\left(b_{j}\right)_{Q}\right) f_{2}\right)(y)\right\| \\
& =H_{1}(x)+H_{2}(x)+H_{3}(x)+H_{4}(x) .
\end{aligned}
$$


For $H_{1}(x)$, we take $1<p<n / \delta, 1 / s=1 / r-\delta / n$. By Hölder's inequality and Lemma 1,2 , we have

$$
\begin{aligned}
& \frac{1}{|Q|} \int_{Q} H_{1}(x) d x \\
& \leq\left(\frac{1}{|Q|} \int_{Q}\left|\prod_{j=1}^{m}\left(b_{j}(x)-\left(b_{j}\right)_{Q}\right)\right|^{q^{\prime}} d x\right)^{1 / q^{\prime}}\left(\frac{1}{|Q|} \int_{Q}\left|S_{\delta}(f)(x)\right|^{q} d x\right)^{1 / q} \\
& \leq C\|\vec{b}\|_{B M O}|Q|^{-1 / q}\left(\int_{Q}|f(x)|^{p} d x\right)^{1 / p} \\
& \leq C\|\vec{b}\|_{B M O} d^{-n(1 / p-\delta / n)}\left\|f \chi_{Q}\right\|_{L^{p}} \\
& \leq C\|\vec{b}\|_{B M O}\|f\|_{B_{p}^{\delta}} .
\end{aligned}
$$

For $H_{2}(x)$, taking $1<p<n / \delta, 1 / s=1 / r-\delta / n$, and $1 / s^{\prime}+1 / s=1$, we obtain that

$$
\begin{aligned}
& \frac{1}{|Q|} \int_{Q} H_{2}(x) d x \\
& \leq C \sum_{j=1}^{m-1} \sum_{\sigma \in C_{j}^{m}}\left(\frac{1}{|Q|} \int_{Q}\left|\left(\vec{b}(x)-\vec{b}_{Q}\right)_{\sigma}\right|^{s^{\prime}} d x\right)^{1 / s^{\prime}} \\
& \left.\qquad\left.\left(\frac{1}{|Q|} \int_{Q} \mid S_{\delta}\left(\left(\vec{b}-\vec{b}_{Q}\right)_{\sigma^{c}}\right) f\right)(x)\right|^{s} d x\right)^{1 / s} \\
& \left.\leq\left. C \sum_{j=1}^{m-1}\left\|\vec{b}_{\sigma}\right\|_{B M O}|Q|^{-1 / s}\left(\int_{R^{n}} \mid\left(b \overrightarrow{(x)}-\vec{b}_{Q}\right)_{\sigma^{c}}\right) f(x)\right|^{r} \chi_{Q} d x\right)^{1 / r} \\
& \leq C \sum_{j=1}^{m-1} \sum_{\sigma \in C_{j}^{m}}\left\|\vec{b}_{\sigma}\right\|_{B M O}\left(\frac{1}{|Q|} \int_{Q}\left|\left(\vec{b}(x)-\vec{b}_{Q}\right)_{\sigma^{c}}\right|^{p r /(p-r)} d x\right)^{(p-r) / p r} \\
& \leq C \sum_{j=1}^{m-1} \sum_{\sigma \in C_{j}^{m}}\left\|\vec{b}_{\sigma}\right\|_{B M O}\left\|\vec{b}_{\sigma^{c}}\right\|_{B M O}(\delta / n-1 / p)\left\|f d^{-n(1 / p-\delta / n)}\right\| f \chi_{Q} \|_{L^{p}} \\
& \leq C|| \vec{b}\left\|_{B M O}\right\| f \|_{B_{D}^{\delta} .}
\end{aligned}
$$

For $H_{3}(x)$, taking $1<p<n / \delta, 1 / s=1 / r-\delta / n$ and $1 / s^{\prime}+1 / s=1$, we get

$$
\frac{1}{|Q|} \int_{Q} H_{3}(x) d x
$$




$$
\begin{aligned}
& \leq\left(\frac{1}{|Q|} \int_{Q}\left|\mu_{\delta}\left(\left(b_{1}-\left(b_{1}\right)_{Q}\right) \cdots\left(b_{m}-\left(b_{m}\right)_{Q}\right) f_{1}\right)(x)\right|^{s} d x\right)^{1 / s} \\
& \leq C|Q|^{-1 / s} \|\left(\left(b_{1}(x)-\left(b_{1}\right)_{Q}\right) \cdots\left(b_{m}(x)-\left(b_{m}\right)_{Q}\right) f \chi_{Q} \|_{L^{r}}\right. \\
& \leq C\left(\frac{1}{|Q|} \int_{Q}\left|\left(b_{1}-\left(b_{1}\right)_{Q}\right) \cdots\left(b_{m}-\left(b_{m}\right)_{Q}\right)\right|^{p r /(p-r)} d x\right)^{(p-r) / p r} \\
& \quad d^{-n(1 / p-\delta / n)}\left\|f \chi_{Q}\right\|_{L^{p}} \\
& \leq C\|\vec{b}\|_{B M O}\|f\|_{B_{p}^{\delta} .} .
\end{aligned}
$$

For $H_{4}(x)$, we have

$$
\begin{aligned}
& H_{4}(x) \\
& \leq\left[\int \int _ { R _ { + } ^ { n + 1 } } \left(\int_{Q^{c}}\left|\chi_{\Gamma(x)}-\chi_{\Gamma\left(x_{0}\right)}\right|\right.\right. \\
& \left.\left.\prod_{j=1}^{m}\left|b_{j}(z)-\left(b_{j}\right)_{Q}\left\|\psi_{t}(y-z)\right\| f(z)\right| d z\right)^{2} \frac{d y d t}{t^{n+1}}\right]^{1 / 2} \\
& \leq C \sum_{k=0}^{\infty} \int_{2^{k+1} Q \backslash 2^{k} Q}\left|x_{0}-x\right|^{1 / 2}\left|x_{0}-z\right|^{-(n+1 / 2-\delta)}\left|\prod_{j=1}^{m}\left(b_{j}(z)-\left(b_{j}\right)_{Q}\right)\right||f(z)| d z \\
& \leq C \sum_{k=1}^{\infty} 2^{-k / 2} \frac{1}{\left|2^{k} Q\right|^{1-\delta / n}} \int_{2^{k} Q}\left|\prod_{j=1}^{m}\left(b_{j}(y)-\left(b_{j}\right)_{2 Q}\right)\right||f(y)| d y \\
& \leq C \sum_{k=1}^{\infty} 2^{-k / 2} \frac{1}{\left|2^{k} Q\right|^{1-\delta / n}}\left(\int_{2^{k} Q}\left|\prod_{j=1}^{m}\left(b_{j}(y)-\left(b_{j}\right)_{2 Q}\right)\right|^{p /(p-1)} d y\right)^{(p-1) / p} \\
& \times\left(\int_{2^{k} Q}|f(y)|^{p} d y\right)^{1 / p} \\
& \leq C \sum_{k=1}^{\infty} 2^{-k / 2}\left(\frac{1}{\left|2^{k} Q\right|} \int_{2^{k} Q}\left|\prod_{j=1}^{m}\left(b_{j}(y)-\left(b_{j}\right)_{2 Q}\right)\right|^{p /(p-1)} d y\right)^{(p-1) / p} \\
& \times\left|2^{k} Q\right|^{-(1 / p-\delta / n)}\left\|f \chi_{2^{k}} Q\right\|_{L^{p}} \\
& \leq C\|\vec{b}\|_{B M O}\|f\|_{B_{p}^{\delta}} .
\end{aligned}
$$

This completes the proof of Theorem 2 . 
Theorem 3. Let $0<\delta<n$ and $\vec{b}=\left(b_{1}, \cdots, b_{m}\right)$ with $b_{j} \in B M O\left(R^{n}\right)$ for $1 \leq j \leq$ $m$. Assume that the following inequality holds for any $H^{1}\left(R^{n}\right)$-atom a supported on a certain cube $Q$ and for $u \in Q$.

$$
\begin{gathered}
\sum_{j=1}^{m} \sum_{\sigma \in C_{j}^{m}} \int_{(2 Q)^{c}}\left[\left|\left(b(x)-b_{Q}\right)_{\sigma^{c}}\right|\right. \\
\left.\left(\iint_{\Gamma(x)}\left(\int_{Q}\left|\left(b(z)-b_{Q}\right)_{\sigma} \psi_{t}(y-u) a(z)\right| d z\right)^{2} \frac{d y d t}{t^{n+1}}\right)^{1 / 2}\right]^{n /(n-\delta)} d x \\
\leq C
\end{gathered}
$$

Then $S_{\delta}^{\vec{b}}$ is bounded from $H^{1}\left(R^{n}\right)$ to $L^{n /(n-\delta)}\left(R^{n}\right)$.

Proof of Theorem 3. Let $a$ be an atom supported in some cube $Q$ and $u \in Q$. We write

$$
\begin{gathered}
\int_{R^{n}}\left|S_{\delta}^{\vec{b}}(a)(x)\right|^{n /(n-\delta)} d x \\
=\int_{2 Q}\left|S_{\delta}^{\vec{b}}(a)(x)\right|^{n /(n-\delta)} d x+\int_{(2 Q)^{c}}\left|S_{\delta}^{\vec{b}}(a)(x)\right|^{n /(n-\delta)} d x=I+I I .
\end{gathered}
$$

For $I$, take $1<p<n / \delta$ and $1 / q=1 / p-\delta / n$. Then we have

$$
I \leq\left\|\mu_{\delta}^{\vec{b}}(a)\right\|_{L^{q}}^{n /(n-\delta)}|2 Q|^{1-n /((n-\delta) q)} \leq C|| a \|_{L^{p}}^{n /(n-\delta)}|Q|^{1-n /((n-\delta) q)} \leq C .
$$

For $I I$, we first calculate $F_{t}^{\vec{b}}(a)(x)$. When $m=1$, we have

$$
\begin{aligned}
\left|F_{t}^{b_{1}}(a)(x, y)\right| & \leq\left|\int_{Q} \psi_{t}(y-z) a(z)\left(b_{1}(x)-\left(b_{1}\right)_{Q}\right) d z\right| \\
& +\left|\int_{Q}\left(\psi_{t}(y-z)-\psi_{t}(y-u)\right) a(z)\left(b_{1}(z)-\left(b_{1}\right)_{Q}\right) d z\right| \\
& +\left|\int_{Q} \psi_{t}(y-u)\left(b_{1}(z)-\left(b_{1}\right)_{Q}\right) a(z) d z\right| \\
& =v_{1}^{\prime}+v_{2}^{\prime}+v_{3}^{\prime},
\end{aligned}
$$

so

$$
\begin{gathered}
S_{\delta}^{b_{1}}(a)(x)=\left\|\chi_{\Gamma(x)} F_{t}^{b_{1}}(a)(x, y)\right\| \\
\leq\left(\iint_{\Gamma(x)}\left|v_{1}^{\prime}\right|^{2} \frac{d y d t}{t^{n+1}}\right)^{1 / 2}+\left(\iint_{\Gamma(x)}\left|v_{2}^{\prime}\right|^{2} \frac{d y d t}{t^{n+1}}\right)^{1 / 2} \\
+\left(\iint_{\Gamma(x)}\left|v_{3}^{\prime}\right|^{2} \frac{d y d t}{t^{n+1}}\right)^{1 / 2}
\end{gathered}
$$




$$
=A^{\prime}(x)+B^{\prime}(x)+C^{\prime}(x) .
$$

For $A^{\prime}(x)$, we have

$$
A^{\prime}(x) \leq S_{\delta}(a)(x)\left|b_{1}(x)-\left(b_{1}\right)_{Q}\right|,
$$

thus

$$
\begin{gathered}
\left(\int_{(2 Q)^{c}}\left(A^{\prime}(x)\right)^{n /(n-\delta)} d x\right)^{(n-\delta) / n} \\
=\left(\int _ { ( 2 Q ) ^ { c } } \left[\left|b_{1}(x)-\left(b_{1}\right) Q\right|\right.\right. \\
\left.\left.\times\left(\iint_{\Gamma(x)}\left|\int_{Q}\left(\psi_{t}(y-z)-\psi_{t}(y-u)\right) a(z) d z\right|^{2} \frac{d y d t}{t^{n+1}}\right)^{1 / 2}\right]^{n /(n-\delta)} d x\right)^{(n-\delta) / n} \\
\leq\left. C|Q|^{1+\varepsilon / n}|| a\right|_{L^{\infty}} \\
\sum_{k=1}^{\infty}\left(\int_{2^{k+1} Q}\left(\frac{\left|2^{k} Q\right|^{\delta / n}}{\left|2^{k} Q\right|^{(n+\varepsilon) / n}}\left|b_{1}(x)-\left(b_{1}\right)_{Q}\right|\right)^{n /(n-\delta)} d x\right)^{(n-\delta) / n} \\
\leq C \sum_{k=1}^{\infty} 2^{-k \varepsilon}\left(\frac{1}{\left|2^{k+1} Q\right|} \int_{2^{k+1} Q} \leq b_{1}(x)-\left.\left(b_{1}\right) Q\right|^{n /(n-\delta)} d x\right)^{(n-\delta) / n} \\
\leq\left. C\left|b_{1}\right|\right|_{B M O} .
\end{gathered}
$$

For $B^{\prime}(x)$, we have

$$
\begin{gathered}
\leq C\left(\iint_{\Gamma(x)}\left(\int_{Q} \frac{B^{\prime}(x)}{(t+|y-u|)^{n+1+\varepsilon-\delta}}|a(z)|\left|b(z)-\left(b_{1}\right)_{Q}\right| d z\right)^{2} \frac{d y d t}{t^{n+1}}\right)^{1 / 2} \\
\leq C\left(\int_{0}^{\infty} \frac{t d t}{(t+|x-u|)^{2(n+1+\varepsilon-\delta)}}\right)^{1 / 2} \int_{Q}|u-z|^{\varepsilon}|a(z)|\left|b_{1}(z)-\left(b_{1}\right)_{Q}\right| d z \\
\leq C|| b_{1}||_{B M O}|x-u|^{-(n+\varepsilon-\delta)}|Q|^{1+\varepsilon / n}|| a \|_{L^{\infty}},
\end{gathered}
$$

thus

$$
\begin{aligned}
& \left(\int_{(2 Q)^{c}}\left(B^{\prime}(x)\right)^{n /(n-\delta)} d x\right)^{(n-\delta) / n} \\
& \leq C\left\|b_{1}\right\|_{B M O}\|a\|_{L^{\infty}}\left[\sum_{k=1}^{\infty} \int_{2^{k+1} Q \backslash 2^{k} Q}\left(\frac{|Q|^{1+\varepsilon / n}}{|x-u|^{n+\varepsilon-\delta}}\right)^{n /(n-\delta)} d x\right]^{(n-\delta) / n}
\end{aligned}
$$


$\leq C \mid\left\|b_{1}\right\|_{B M O} \sum_{k=1}^{\infty} 2^{-k \varepsilon}$

$\leq C \mid\left\|b_{1}\right\|_{B M O}$.

From that we know, if

$$
\begin{aligned}
& \int_{(2 Q)^{c}}\left(C^{\prime}(x)\right)^{n /(n-\delta)} d x \\
& =\int_{(2 Q)^{c}}\left[\left(\int \int _ { \Gamma ( x ) } \left(\int_{Q} \mid\left(b_{1}(z)-\left(b_{1}\right)_{Q}\right)\right.\right.\right. \\
& \left.\left.\left.\psi_{t}(y-u) a(z) \mid d z\right)^{2} \frac{d y d t}{t^{n+1}}\right)^{1 / 2}\right]^{n /(n-\delta)} d x \leq C,
\end{aligned}
$$

then

$$
\int_{R^{n}}\left|S_{\delta}^{b_{1}}(a)(x)\right|^{n /(n-\delta)} d x \leq C
$$

When $m>1$, we have

$$
\begin{gathered}
\left|F_{t}^{\vec{b}}(a)(x, y)\right| \leq\left|\prod_{j=1}^{m}\left(b_{j}(x)-\left(b_{j}\right)_{Q}\right) \int_{Q} \psi_{t}(y-z) a(z) d z\right| \\
+\sum_{j=1}^{m} \sum_{\sigma \in C_{j}^{m}}\left|\left(\vec{b}(x)-\vec{b}_{Q}\right)_{\sigma^{c}} \int_{Q}\left(\psi_{t}(y-z)-\psi_{t}(y-u)\right)\left(\vec{b}(z)-\vec{b}_{Q}\right)_{\sigma} a(z) d z\right| \\
+\sum_{j=1}^{m} \sum_{\sigma \in C_{j}^{m}}\left|\left(\vec{b}(x)-\vec{b}_{Q}\right)_{\sigma^{c}} \int_{Q} \psi_{t}(y-u)\left(\vec{b}(z)-\vec{b}_{Q}\right)_{\sigma} a(z) d z\right| \\
=v_{1}+v_{2}+v_{3},
\end{gathered}
$$

so

$$
\begin{gathered}
S_{\delta}^{\vec{b}}(a)(x)=\left\|\chi_{\Gamma(x)} F_{t}^{\vec{b}}(a)(x, y)\right\| \\
\leq\left(\iint_{\Gamma(x)}\left|\nu_{1}\right|^{2} \frac{d y d t}{t^{n+1}}\right)^{1 / 2}+\left(\iint_{\Gamma(x)}\left|\nu_{2}\right|^{2} \frac{d y d t}{t^{n+1}}\right)^{1 / 2} \\
+\left(\iint_{\Gamma(x)}\left|\nu_{3}\right|^{2} \frac{d y d t}{t^{n+1}}\right)^{1 / 2} \\
=A(x)+B(x)+C(x) .
\end{gathered}
$$


For $A(x)$, we have

$$
\begin{aligned}
A(x) & =\left(\iint_{\Gamma(x)} \prod_{j=1}^{m}\left|b_{j}(x)-\left(b_{j}\right)_{Q}\right|^{2}\left|\int_{Q} \psi_{t}(y-z) a(z) d z\right|^{2} \frac{d y d t}{t^{n+1}}\right)^{1 / 2} \\
& =\prod_{j=1}^{m}\left|b_{j}(x)-\left(b_{j}\right)_{Q}\right| S_{\delta}(a)(x)
\end{aligned}
$$

thus

$$
\begin{aligned}
& \left(\int_{(2 Q)^{c}}(A(x))^{n /(n-\delta)} d x\right)^{(n-\delta) / n} \\
& \leq C\|a\|_{L^{\infty}} \\
& {\left[\sum_{k=1}^{\infty} \int_{2^{k+1} Q \backslash 2^{k} Q}\left(\frac{|Q|^{1+\varepsilon / n}}{|x-u|^{n+\varepsilon-\delta}} \prod_{j=1}^{m}\left|b_{j}(x)-\left(b_{j}\right)_{Q}\right|\right)^{n /(n-\delta)} d x\right]^{(n-\delta) / n}} \\
& \leq C|Q|^{1+\varepsilon / n}|| a \|_{L^{\infty}} \\
& \sum_{k=1}^{\infty}\left(\int_{2^{k+1} Q}\left(\frac{\left|2^{k} Q\right|^{\delta / n}}{\left|2^{k} Q\right|^{(n+\varepsilon) / n}} \prod_{j=1}^{m}\left|b_{j}(x)-\left(b_{j}\right)_{Q}\right|\right)^{n /(n-\delta)} d x\right)^{(n-\delta) / n} \\
& \leq C \sum_{k=1}^{\infty} 2^{-k \varepsilon}\left(\frac{1}{\left|2^{k+1} Q\right|} \int_{2^{k+1} Q}\left(\prod_{j=1}^{m}\left|b_{j}(x)-\left(b_{j}\right)_{Q}\right|\right)^{n /(n-\delta)} d x\right)^{(n-\delta) / n} \\
& \leq C\|\vec{b}\|_{B M O} .
\end{aligned}
$$

For $B(x)$, we have

$$
\begin{aligned}
B(x) & =\left(\iint_{\Gamma(x)} \mid \sum_{j=1}^{m} \sum_{\sigma \in C_{j}^{m}}\left(\vec{b}(x)-\vec{b}_{Q}\right)_{\sigma^{c}}\right. \\
& \left.\times\left.\int_{Q}\left(\psi_{t}(y-z)-\psi_{t}(y-u)\right)\left(\vec{b}(z)-\vec{b}_{Q}\right)_{\sigma} a(z) d z\right|^{2} \frac{d y d t}{t^{n+1}}\right)^{1 / 2} \\
& \leq C \sum_{j=1}^{m} \sum_{\sigma \in C_{j}^{m}}\left|\left(\vec{b}(x)-\vec{b}_{Q}\right)_{\sigma^{c}}\right| \\
& \times\left(\iint_{\Gamma(x)}\left(\int_{Q} \frac{t|u-z|^{\varepsilon}}{(t+|y-u|)^{n+1+\varepsilon-\delta}}\left|\left(\vec{b}(z)-\vec{b}_{Q}\right)_{\sigma}\right||a(z)| d z\right)^{2} \frac{d y d t}{t^{n+1}}\right)^{1 / 2}
\end{aligned}
$$




$$
\begin{aligned}
& \leq C \sum_{j=1}^{m} \sum_{\sigma \in C_{j}^{m}}\left|\left(\vec{b}(x)-b_{Q}\right)_{\sigma^{c}}\right|\left(\iint_{\Gamma(x)} \frac{t^{1-n} d y d t}{(t+|y-u|)^{2(n+1+\varepsilon-\delta)}}\right)^{1 / 2} \\
& \times \int_{Q}|u-z|^{\varepsilon}\left|a(z) \|\left(\vec{b}(z)-\vec{b}_{Q}\right)_{\sigma}\right| d z \\
& \leq C \sum_{j=1}^{m} \sum_{\sigma \in C_{j}^{m}}\left|\left(\vec{b}(x)-\vec{b}_{Q}\right)_{\sigma^{c}}\right||x-u|^{-(n+\varepsilon)}|Q|^{1+\varepsilon / n-\delta}|| a\left\|_{L^{\infty}}\right\| \vec{b}_{\sigma} \|_{B M O},
\end{aligned}
$$

thus

$$
\begin{gathered}
\left(\int_{(2 Q)^{c}}(B(x))^{n /(n-\delta)} d x\right)^{(n-\delta) / n} \\
\leq C \sum_{j=1}^{m} \sum_{\sigma \in C_{j}^{m}} \sum_{k=1}^{\infty} 2^{-k \varepsilon} \\
\left(\frac{1}{\left|2^{k+1} Q\right|} \int_{2^{k+1} Q}\left|(\vec{b}(x)-\vec{b} Q)_{\sigma^{c}}\right|^{n /(n-\delta)} d x\right)^{(n-\delta) / n}\left\|\vec{b}_{\sigma}\right\|_{B M O} \\
\leq C \mid \vec{b} \|_{B M O} .
\end{gathered}
$$

So, if

$$
\begin{gathered}
\int_{(2 Q)^{c}}(C(x))^{n /(n-\delta)} d x \\
=\sum_{j=1}^{m} \sum_{\sigma \in C_{j}^{m}} \int_{(2 Q)^{c}}\left[| ( b ( x ) - b _ { Q } ) _ { \sigma ^ { c } } | \left(\int \int _ { \Gamma ( x ) } \left(\int_{Q} \mid\left(b(z)-b_{Q}\right)_{\sigma}\right.\right.\right. \\
\left.\left.\left.\psi_{t}(y-u) a(z) \mid d z\right)^{2} \frac{d y d t}{t^{n+1}}\right)^{1 / 2}\right]^{n /(n-\delta)} d x \\
\leq C,
\end{gathered}
$$

then

$$
\int_{R^{n}}\left|S_{\delta}^{\vec{b}}(a)(x)\right|^{n /(n-\delta)} d x \leq C .
$$

This completes the proof of Theorem 3.

\section{REFERENCES}

[1] J. Alvarez, R. J. Bagby, D. S. Kurtz, and C. Pérez, "Weighted estimates for commutators of linear operators," Studia Math., vol. 104, no. 2, pp. 195-209, 1993.

[2] W. Chen and G. Hu, "Weak type $\left(H^{1}, L^{1}\right)$ estimate for a multilinear singular integral operator," Adv. Math. (China), vol. 30, no. 1, pp. 63-69, 2001. 
[3] R. R. Coifman, R. Rochberg, and G. Weiss, "Factorization theorems for Hardy spaces in several variables," Ann. of Math. (2), vol. 103, no. 2, pp. 611-635, 1976.

[4] J. García-Cuerva and J. L. Rubio de Francia, Weighted norm inequalities and related topics, ser. North-Holland Math. Stud. Amsterdam: North-Holland Publishing Co., 1985, vol. 116.

[5] E. Harboure, C. Segovia, and J. L. Torrea, "Boundedness of commutators of fractional and singular integrals for the extreme values of p," Illinois J. Math., vol. 41, no. 4, pp. 676-700, 1997.

[6] L. Liu, "Weighted weak type $\left(H^{1}, L^{1}\right)$ estimates for commutators of Littlewood-Paley operators," Indian J. Math., vol. 45, no. 1, pp. 71-78, 2003.

[7] L. Liu, "Weighted block- $H^{1}$ estimates for commutators of Littlewood-Paley operators," Southeast Asian Bull. Math., vol. 27, no. 5, pp. 833-838, 2004.

[8] C. Pérez and R. Trujillo-Gonzq'alez, "Sharp weighted estimates for multilinear commutators," $J$. London Math. Soc. (2), vol. 65, no. 3, pp. 672-692, 2002.

[9] E. M. Stein, Harmonic analysis: real-variable methods, orthogonality, and oscillatory integrals, ser. Princeton Math. Ser. Princeton, NJ: Princeton University Press, 1993, vol. 43.

[10] A. Torchinsky, Real-variable methods in harmonic analysis, ser. Pure Appl. Math. Orlando, FL: Academic Press, Inc., 1986, vol. 123.

\section{Author's address}

\section{Jiasheng Zeng}

Hunan Business College, Department of Mathematics, Changsha, 410205, P. R. of China

E-mail address: zengjiashenga@163.com 